\title{
Model Hydrology MockWyn-UB to Analyse Water Availability in Gumbasa Watershed Central Sulawesi Province
}

\author{
A. Solihin Ansari ${ }^{1}$ I Wayan Sutapa ${ }^{2}$, M. Galib Ishak ${ }^{3}$ \\ ${ }^{1}$ Program Master Civil Engineering, University of Tadulako, Central Sulawesi, Indonesia \\ ${ }^{2,3}$ Department of Civil Engineering, University of Tadulako, Cenral Sulawesi, Indonesia
}

\begin{abstract}
From the results of previous studies stating that the watershed Gumbasa there has been a change in climate, discharge models used in calculating the availability of water in a watershed used the model MockWyn-UB Studies conducted Gu mbasa watershed has an area of $1229.43 \mathrm{~km}^{2}$ with AWLR outlet Gu mbasa River. Based on the analysis, the relationship between the discharge of models and the discharge observation forming a un iform pattern except in 2013 which discharge higher models of discharge observation. The correlation coefficient between the discharge and the discharge observation models do not qualify so the calibration data using the facility solver is not done. Thus the calibration is done using the Root Mean Square Error (RMSE). RMSE value MockWyn-UB acquired 3.10\%. While the results of the analysis models Mock RMSE values obtained 8.03 $\%$.Comparison between the using of model FJ. Mock and model MockWyn-UB with using test parameters RMSE statistics showed an error rate MockWyn-UB models better than models FJ. Mock. Model MockWynUB do not optimal if applied in the watershed Gumbasa, this is caused by the presence of Lake of Lindu are located in the basin Gumbasa.

Keywords: FJ. Mock, Gumbasa Watershed, Lake Lindu, MockWyn UB, RMSE.
\end{abstract}

\section{INTRUDUCTION}

The condition of the rainy season and the dry season in the last few years is no longer in accordance with the conditions that existed before. This is influenced by the existence of global warming due to greenhouse effect so that the air temperature of the earth has increased significantly, this phenomenon is called climate change.With the climate change, will affect the hydrological processes that occur in the watershed. Gumbasa watershed is part of the Palu watershed area of $1229.43 \mathrm{~km}^{2}$ has tipped in Masomba mountain, Mount Nokilalaki and Lake of Lindu. With a large area Watershed Gumbasa also do not immune from climate change. Given that climate change will certainly affect the availability of water and hydrologic conditions that occurred in the river basin. When this has been a lot of modeling discharges that have been found by Hydrologists including model of FJ. Mock 1973[1], NRECA Model[2],Model Nugroho[3], Model MockWyn-UB [4] of the many modeling discharge mentioned above, only one model that incorporate climate change parameters in calculating the availability of water in a river basin. That is the model MockWynUB. This discharge models by Sutapa, I.Wayan [4] is a model of the balance of water / rain water flow balance climate simulations based on development of the model FJ. Mock [1] by the model name MockWyn-UB. This research was carried out by inserting a natural phenomenon that occurs at this time these as a novelty in this study such as climate change, canopy interception, rainfall distribution based on land use, soil type and soil characteristics. Model Mockwyn-UB a new debit calculation d.To prove the reliability it needs to be applied in other watersheds, which has wide and different characteristics of the watershed Previous.

\section{RESEARCH METHODS}

The location this research lies in the watershed of Gumbasa is part of the watershed area of Palu have $1229,43 \mathrm{~km}^{2}$ at Masomba mount, Nokilalaki mount dan Lake Lindu. Geographically situated the River watershed Gumbasa between $01^{0}$ $01^{\prime} \mathrm{LS}-01^{0} 21^{\prime} \mathrm{LS}$ and $119^{0} 56^{\prime} \mathrm{BT}-120^{\circ} 19^{\prime} \mathrm{BT}$. The location study can be seen in the map below: 


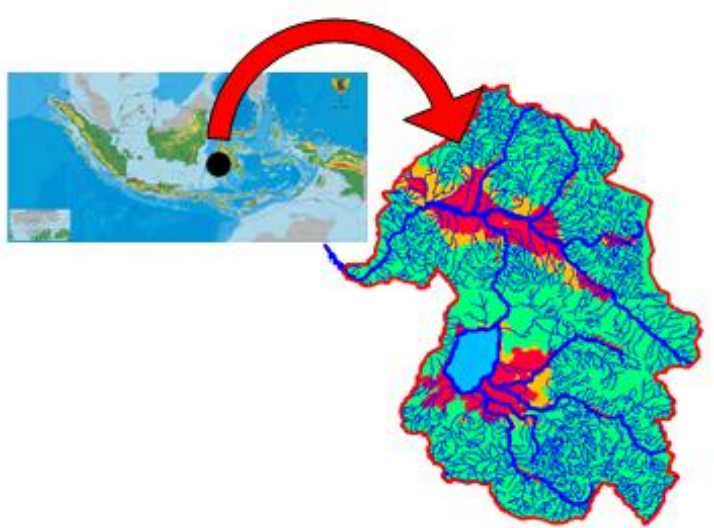

Figure 1. Location of research

The type of data used in this study consisted of primary data and secondary data. The primary data in the form of soil samples doing in Kapiroe Village, Village Tongoa and Sintuwu, Secondary data consists of: 1) the data of precipitation at the station Bora, Bangga Low, Kulawi, Palolo, Wuasa (2002-2015); 2) data of climatology at stations Bora (2002-2015); 3) data of discharge Gumbasa river; 4) map of the earth Indonesia; 5) map of land use (2015). Data collection techniques in this research refers to research that has been done by I Wayan Sutapa (2013).

The data used as input in the modeling of the discharge was analyzed in the following methods:

a. Detection of the presence or absence of climate trends and make projections of climate change. In this study the above step, not done because previous research has shown that in the region there has been a climate change.

b. Analysis of average rainfall watershed using methods Theissen $[5,6]$ caused rainfall data is not uniform, the calculate of rain continued net based with land cover using an research dunne and Leopold [7]

c. Calculate potential evapotranspiration with using the Penman Monteith [8]

d. The calculated water balanced with use model of MockWyn UB[4]

e. Result calibration model the discharge of MockWyn UB with data AWLR at Gumbasa river, the using of method of statistic Root Mean Square Error[9]

The tank model of MockWyn UB can be seen in Figure 2.

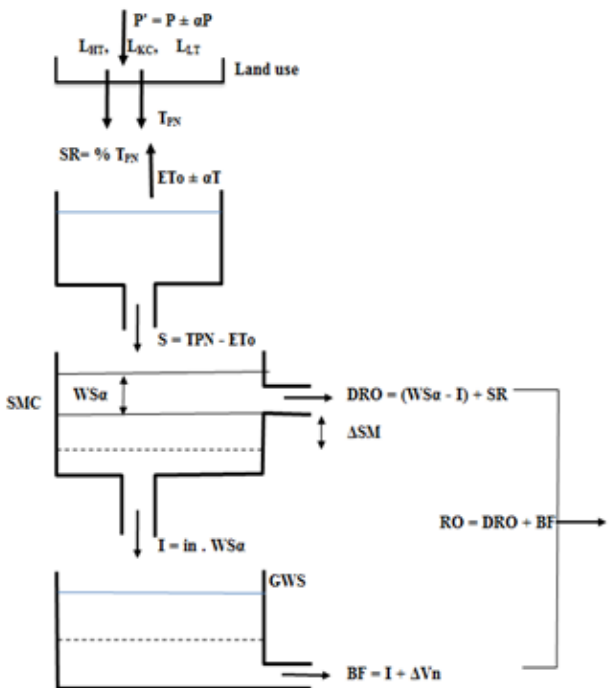

Figure 2. Tank model of MockWyn UB[1]

III. RESULT AND DISCUSSION

3.1 Deposits of groundwater
The primary data in the form of soil samples and vegetation based on the existing land 
is: open land, gardens and forests mix. Soil sampling was conducted on March 27, 2016 in the village Kapiroe, villages Tongoa and Sintuwu. Soil samples are then analyzed in Soil Mechanics
Laboratory University of Tadulako to determine the soil moisture content at each layer of soil. Groundwater levels are described in Table 1

Table 1. Water content of soil

\begin{tabular}{|c|c|c|c|c|c|c|c|}
\hline No & $\begin{array}{l}\text { Location of } \\
\text { Sample }\end{array}$ & Subsoil & $\begin{array}{l}\text { Thick } \\
\text { (mm) }\end{array}$ & $\begin{array}{l}\text { Water } \\
\text { content } \\
(\%)\end{array}$ & $\begin{array}{l}\text { Content } \\
\text { weight of soil } \\
\left(\mathrm{gr} / \mathrm{cm}^{3}\right)\end{array}$ & $\begin{array}{l}\text { Water content } \\
\text { volumetric }(\%)\end{array}$ & $\begin{array}{l}\text { Thick of water } \\
\text { content (mm) }\end{array}$ \\
\hline \multirow[t]{4}{*}{1} & \multirow{4}{*}{$\begin{array}{ll}\text { TP } & 01 \\
\text { (garden) } & \\
\text { Village } & \text { of } \\
\text { Sintuwu } & \end{array}$} & Layer of surface & 150 & 23.26 & 1151 & 26.77 & 40.16 \\
\hline & & Layer of root & 650 & 20.59 & 1440 & 29.65 & 192.72 \\
\hline & & Layer of moist & 500 & 9.23 & 1514 & 13.97 & 69.87 \\
\hline & & & & & & Average & 199.92 \\
\hline \multirow[t]{4}{*}{2} & \multirow{4}{*}{$\begin{array}{l}\text { TP } \\
\text { (Forest) } \\
\text { Village } \\
\text { Tongoa }\end{array}$} & Layer of surface & 150 & 16.20 & 1250 & 20.25 & 30.37 \\
\hline & & Layer of root & 650 & 9.85 & 1544 & 15.21 & 98.85 \\
\hline & & Layer of moist & 500 & 8.95 & 1682 & 15.05 & 75.27 \\
\hline & & & & & & Average & 68.16 \\
\hline \multirow[t]{5}{*}{3} & \multirow{4}{*}{$\begin{array}{l}\text { TP 03 (Land } \\
\text { open) } \\
\text { Village of } \\
\text { Kapiroe }\end{array}$} & Layer of surface & 150 & 25.37 & 1721 & 43.66 & 43.66 \\
\hline & & Layer of root & 650 & 22.34 & 1672 & 37.35 & 186.76 \\
\hline & & Layer of moist & 1000 & 25.78 & 1779 & 45.86 & 458.62 \\
\hline & & & & & & Average & 229.68 \\
\hline & & & & & & Average & 132.92 \\
\hline
\end{tabular}

The results of analysis initial deposit groundwater is $45.56 \mathrm{~mm}$, while the maximum water savings is calculated using the method of De Laat[10]be obtained $186.67 \mathrm{~mm}$.
The method used to calculate the average rainfall is a method of Thiessen[5,6], this method is used if the spread of rainfall stations in areas of interest uneven. Data from the average rainfall can be seen in the table 2

\subsection{Aver age rainfall}

Table 2. Average of Precipitation

\begin{tabular}{|l|l|l|l|l|l|l|l|l|l|l|l|l|l|}
\hline \multirow{2}{*}{ No } & \multirow{2}{*}{ Month } & \multicolumn{3}{|l|}{ Average Rainfall Data Year 2002 (Station) } & \multicolumn{3}{l|}{ Precipitation Method of Thiessen (Station) } & Precipitation \\
\cline { 2 - 14 } & & Bora & Kulawi & Palolo & Banga B & Wuasa & Bora & Kulawi & Palolo & Bangga B & Wuasa & Region (mm ) \\
\hline 1 & January & 110.00 & 133.10 & 9.80 & 90.60 & 106.00 & 7513.00 & 42005.029 & 1750.28 & 25343.54 & 22473.06 & 93.99 \\
\hline 2 & February & 9.70 & 34.30 & 0.00 & 24.90 & 75.60 & 662.51 & 10824.737 & 0 & 6965.277 & 16027.96 & 32.71 \\
\hline 3 & March & 44.10 & 336.30 & 71.90 & 3.40 & 243.80 & 3012.03 & 106132.92 & 12841.34 & 951.082 & 51688.04 & 165.64 \\
\hline 4 & April & 0.00 & 435.20 & 211.30 & 0.00 & 125.30 & 0.00 & 137344.77 & 37738.18 & 0 & 26564.85 & 191.27 \\
\hline 5 & May & 145.60 & 193.00 & 84.30 & 42.00 & 124.90 & 9944.48 & 60908.87 & 15055.98 & 11748.66 & 26480.05 & 117.75 \\
\hline 6 & June & 127.00 & 128.10 & 100.30 & 259.50 & 155.90 & 8674.10 & 40427.079 & 17913.58 & 72589.94 & 33052.36 & 163.78 \\
\hline 7 & July & 135.00 & 102.00 & 74.90 & 107.20 & 23.70 & 9220.50 & 32190.18 & 13377.14 & 29987.06 & 5024.637 & 85.18 \\
\hline 8 & August & 20.00 & 61.20 & 119.50 & 36.70 & 55.40 & 1366.00 & 19314.108 & 21342.7 & 10266.09 & 11745.35 & 60.74 \\
\hline 9 & September & 0.00 & 121.20 & 52.60 & 121.80 & 196.20 & 0.00 & 38249.508 & 9394.36 & 34071.11 & 41596.36 & 116.97 \\
\hline 10 & October & 5.00 & 89.40 & 5.90 & 83.40 & 24.50 & 341.50 & 28213.746 & 1053.74 & 23329.48 & 5194.245 & 55.14 \\
\hline 11 & November & 0.00 & 195.60 & 23.00 & 184.30 & 109.40 & 0.00 & 61729.404 & 4107.8 & 51554.24 & 23193.89 & 133.35 \\
\hline 12 & December & 126.80 & 181.70 & 48.20 & 55.30 & 198.80 & 8660.44 & 57342.703 & 8608.52 & 15469.07 & 42147.59 & 125.43 \\
\hline
\end{tabular}

\subsection{Potential e vapotrans piration}

Potential evapotranspiration calculated by Penman Monteith method [8] is based on climatology data Bora station for observation period 2002-2015.

Tabel 3. Evapotranspiration of potential

\begin{tabular}{|c|c|c|c|c|c|c|c|c|c|c|c|c|}
\hline Year & January & February & March & April & May & June & July & August & September & October & November & December \\
\hline \hline $\mathbf{2 0 0 2}$ & 105.41 & 90.70 & 101.96 & 115.78 & 108.19 & 80.81 & 124.85 & 129.91 & 112.76 & 126.85 & 113.66 & 119.41 \\
\hline $\mathbf{2 0 0 3}$ & 99.35 & 87.73 & 95.43 & 100.33 & 111.68 & 115.83 & 94.30 & 92.64 & 97.04 & 38.38 & 110.83 & 47.30 \\
\hline $\mathbf{2 0 0 4}$ & 107.29 & 90.23 & 107.84 & 103.57 & 110.88 & 102.95 & 98.29 & 126.79 & 116.43 & 131.74 & 118.90 & 117.39 \\
\hline $\mathbf{2 0 0 5}$ & 106.28 & 96.57 & 130.84 & 104.59 & 106.91 & 101.99 & 100.42 & 127.74 & 102.38 & 118.24 & 96.49 & 84.18 \\
\hline $\mathbf{2 0 0 6}$ & 98.27 & 99.79 & 110.63 & 103.58 & 119.81 & 93.31 & 115.28 & 121.17 & 114.74 & 124.63 & 111.81 & 108.22 \\
\hline $\mathbf{2 0 0 7}$ & 91.54 & 102.40 & 99.71 & 99.89 & 110.58 & 60.37 & 74.70 & 84.76 & 105.91 & 92.09 & 24.40 & 89.19 \\
\hline
\end{tabular}

Tabel 3. Evapotranspiration of potential (Continued) 


\begin{tabular}{|c|c|c|c|c|c|c|c|c|c|c|c|c|}
\hline Year & January & February & March & April & May & June & July & August & September & October & November & December \\
\hline \hline $\mathbf{2 0 0 8}$ & 101.05 & 80.76 & 91.53 & 89.93 & 64.28 & 33.50 & 35.48 & 37.40 & 39.39 & 42.36 & 39.77 & 40.65 \\
\hline $\mathbf{2 0 0 9}$ & 96.60 & 71.81 & 103.33 & 106.59 & 104.49 & 82.46 & 110.32 & 121.43 & 39.39 & 42.36 & 39.77 & 40.65 \\
\hline $\mathbf{2 0 1 0}$ & 99.82 & 110.53 & 119.58 & 91.27 & 108.25 & 100.74 & 93.49 & 94.02 & 119.08 & 113.09 & 117.15 & 64.65 \\
\hline $\mathbf{2 0 1 1}$ & 100.57 & 72.37 & 86.94 & 90.04 & 107.52 & 97.75 & 88.86 & 113.75 & 103.45 & 125.05 & 108.48 & 91.57 \\
\hline $\mathbf{2 0 1 2}$ & 97.43 & 73.29 & 116.16 & 122.95 & 134.12 & 101.58 & 108.35 & 138.61 & 143.82 & 139.37 & 124.57 & 119.85 \\
\hline $\mathbf{2 0 1 3}$ & 110.72 & 112.22 & 134.38 & 113.70 & 113.57 & 105.76 & 80.05 & 110.57 & 127.01 & 135.50 & 121.03 & 114.47 \\
\hline $\mathbf{2 0 1 4}$ & 80.95 & 158.74 & 135.33 & 126.34 & 113.38 & 99.03 & 133.53 & 124.55 & 145.53 & 156.62 & 120.12 & 41.70 \\
\hline $\mathbf{2 0 1 5}$ & 95.45 & 98.53 & 107.65 & 102.27 & 109.65 & 86.46 & 120.67 & 134.23 & 128.59 & 36.63 & 114.69 & 104.97 \\
\hline
\end{tabular}

\subsection{Discharge of MockWyn UB}

Calculation of net rainfall is the average monthly precipitation fell in each land located in the watershed of Gumbasa.
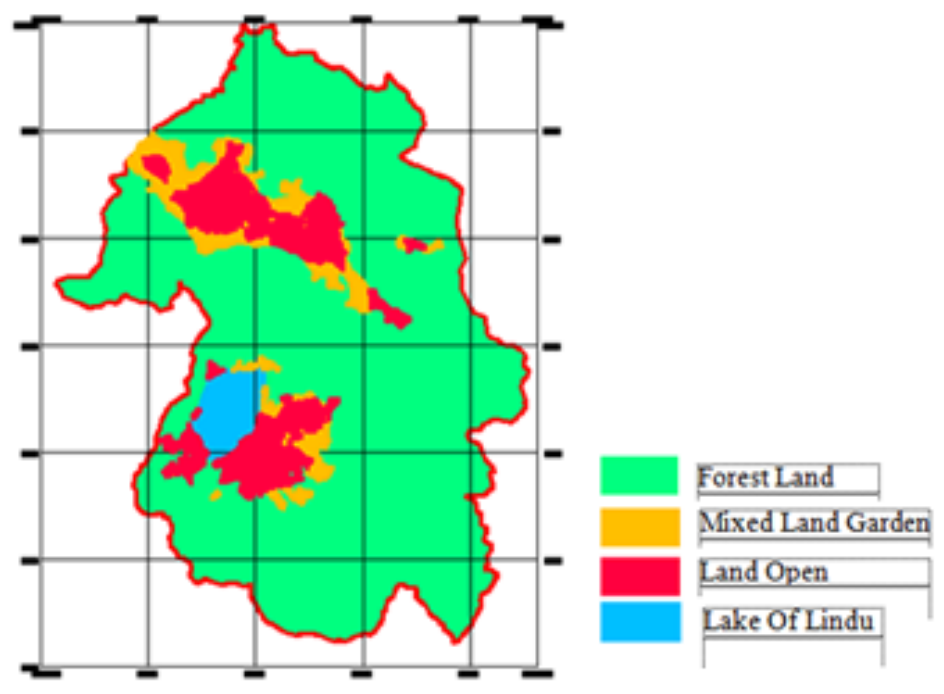

Figure 3. Land use

Table 4. Area of Land Use

\begin{tabular}{|c|l|c|c|}
\hline No & \multicolumn{1}{|c|}{ Land Use } & Unit & Area \\
\hline \hline 1 & Forest land & $\mathrm{Km}^{2}$ & 955.85 \\
\hline 2 & Mixed land Gardens & $\mathrm{Km}^{2}$ & 113.28 \\
\hline 3 & Land Open & $\mathrm{Km}^{2}$ & 103.91 \\
\hline 4 & River and Lake & $\mathrm{Km}^{2}$ & 56.39 \\
\hline \multicolumn{3}{|c|}{ Amount } & $\mathbf{1 2 2 9 . 4 3}$ \\
\hline
\end{tabular}

The results of analysis of net rainfall Gumbasa for watershed areas, so that the flow rate calculation MockWynUB[1] can be done. The simu lation model MockWyn UB models can be seen in Table 5 
Table 5 . Simu lation of discharge model MockWyn UB in Year 2002

\begin{tabular}{|c|c|c|c|c|c|c|c|c|c|c|c|c|c|c|c|c|}
\hline No. & Explanation & Unit & Exp & Jan & Peb & Mar & Apr & May & Jun & Jul & Aug & Sep & Oct & Nov & Des & Year \\
\hline$\overline{\bar{I}}$ & " VEGETATION & & & & & & & & & & & & & & & \\
\hline 1 & Forest land (LHT) & $\mathrm{km}^{2}$ & 955.85 & & & & & & & & & & & & & \\
\hline 2 & Mixed Land gadens (LKC) & $\mathrm{km}^{3}$ & 1138 & 몪 & & & & & & & & & & & & \\
\hline 3 & Land apen (LLT) & $\mathrm{km}^{4}$ & 103. 91 & & & & & & & & & & & & & \\
\hline 4 & Area ofW aters hed (LDAS) & $\mathrm{km}^{5}$ & I 因. 43 & & & & & & & & & & & & & \\
\hline II & Rain \& EVAPOTRANSPIRATION & & & & & & & & & & & & & & & \\
\hline \multirow[t]{2}{*}{5} & The aver age monthly rainfall (P) & $\mathrm{mm} / \mathrm{mth}$ & data & 93. 99 & $32 \pi$ & 165.64 & 191.7 & $117 \pi$ & 163.78 & 85.18 & 60. 74 & 11697 & 55.14 & 133. 35 & $1 \% .43$ & 134. 95 \\
\hline & $\begin{array}{l}\text { The aver age manthly rainfall (P)... } \\
\text { Corrected } \alpha \mathrm{P}\end{array}$ & $\mathrm{mm} / \mathrm{m}$ th & $1 \mathrm{TI}$ & 1127 & 39. $\mathrm{T}$ & 198.7 & 2⿴囗. 53 & 14. 30 & 196.53 & $102 \mathbb{Z}$ & 7289 & 14036 & 66.17 & I60. 02 & $\mid 50.51$ & 161034 \\
\hline 6 & Rain in the Forest (PH) & $\mathrm{mm} / \mathrm{mth}$ & count & 87. 69 & 30.5l & 15454 & 17.45 & 109. 86 & 15280 & 7. 47 & 56. 67 & 109.13 & 51.45 & 18.41 & 11702 & $1 \% 200$ \\
\hline 7 & Rain in the gardes mix ed (РКГ) & $\mathrm{mm} / \mathrm{m}$ th & count & 1039 & 3. 62 & 1831 & 2.15 & 1302 & 1811 & 9. 42 & 6. 72 & 1293 & 6.10 & 1474 & 1387 & 14838 \\
\hline 8 & Rain in the open land (PLT) & $\mathrm{mm} / \mathrm{m}$ th & count & 9. 53 & 3. 32 & 1680 & 1940 & 11. 94 & 16 的 & 8. 64 & 6.16 & 11. 86 & 5.59 & 1353 & 1272 & 136.10 \\
\hline 9 & $\begin{array}{l}\text { Rain net in the forest (PNHT) } \\
=0,886 P+0,088\end{array}$ & $\mathrm{~mm} / \mathrm{mth}$ & count & 77. 78 & 2.12 & 137. 01 & 158. D & 97.42 & 135.47 & 刃. 50 & 50.30 & 的. 70 & 45. 67 & 111032 & 103.7 & 111033 \\
\hline 10 & $\begin{array}{l}\text { Rain net in the gar den mix } \\
(P N K C)=0,925 P+\square, 333\end{array}$ & $\mathrm{~mm} / \mathrm{mth}$ & count & 9. 95 & 3. 68 & 17. 7 & 1990 & 1238 & 17. 08 & 9. 04 & 6. 55 & 1230 & 5.97 & 1397 & 1316 & 14.2 \\
\hline 12 & Total rainfall net (TPN) & $\mathrm{mm} / \mathrm{m}$ th & count & 97. $\mathrm{B}$ & 34.12 & 17. 08 & 197.49 & 12.74 & 169.16 & 88.18 & 63. 00 & 17.93 & 57. 8 & 137.82 & 18.65 & 1387. 68 \\
\hline 13 & $\begin{array}{l}\text { Evapotranspir ation of potencial } \\
\text { (ETo) }\end{array}$ & $\mathrm{mm} / \mathrm{mth}$ & count & 105.4 & 跑 刃 & I01. 96 & 11578 & 108.19 & 80. 81 & 18. 85 & 1891 & $112 \pi$ & 18.85 & 11366 & 11941 & 1330. 30 \\
\hline \multirow[t]{3}{*}{14} & Evapotranspir astion actual (ETA) & & & & & & & & & & & & & & & \\
\hline & TPN > Ela then ETA = ETo & $\mathrm{mm} / \mathrm{mth}$ & count & - & -- & |ㅁ. 96 & 11578 & 108.19 & 80. 81 & - & - & $112 \pi$ & - & 11366 & 1194 & $\pi 257$ \\
\hline & $T P N<E$ o then ETA $=T P N+\triangle S M$ & $\mathrm{~mm} / \mathrm{m}$ th & count & 97. T & 80.84 & - & - & - & - & 12. 47 & 109. 2 & - & 11535 & -- & - & 52.12 \\
\hline III & WATER BALANCED & & & & & & & & & & & & & & & \\
\hline 15 & $\begin{array}{l}\text { The differ ence betw een TPN } \\
W \pi H n \operatorname{ET}(S=T P N-E T a)\end{array}$ & $\mathrm{mm} / \mathrm{mth}$ & $(12-(13)$ & -8.15 & -56.58 & 69.12 & 8. 72 & 1355 & 88.35 & -36.67 & -66.91 & 8.17 & -69.62 & 24. 16 & 108 & \\
\hline $16 \mathrm{a}$ & The loss of potential water & $\mathrm{mm} / \mathrm{mth}$ & count & -8.15 & -56.58 & ०. प्र & ०. प्र & ०. प0 & ०. 0 & -36.67 & -66.91 & ०. प्र & -69.62 & ०. प0 & ०. प्र० & \\
\hline $16 b$ & $\begin{array}{l}\text { Ac cumulation ofpotential w ater } \\
\text { loss (APWL) }\end{array}$ & $\mathrm{mm} / \mathrm{mth}$ & count & -8.15 & -64.73 & ०. प्र & ०. प0 & ०. ०० & ०. प0 & -36.67 & -103.58 & ०. प0 & -69.62 & ०. ०० & ०. प0 & \\
\hline 17 & $\begin{array}{l}\text { Sail maisture SM = SM C. e ^ } \\
\text { (APWL / SM C) }\end{array}$ & $\mathrm{mm} / \mathrm{mth}$ & count & IT. $\pi$ & 131. 97 & 186.67 & 186. 67 & 186.67 & 186. 67 & 153.38 & 107.17 & 186.67 & 18.56 & 186.67 & 186. 67 & \\
\hline 18 & The Cgane of Sail Moisture ( $\Delta S M)$ & $\mathrm{mm} / \mathrm{m}$ th & count & ०. 0 (1) & -46.72 & $54 . \pi$ & ०. प्र & ०. 0 (1) & ०. 0 & -33.8 & -46.7 & T9. 50 & -58.11 & 炽. $\|$ & ०. प्र० & \\
\hline 19 & Deficit $\left(W D=E T_{0}-E A\right)$ & $\mathrm{mm} / \mathrm{m}$ th & (13) - (14) & 8.15 & 9. 85 & - & - & - & - & 3. 38 & ㄱ. $\pi$ & - & 11.5 & -- & - & 53.60 \\
\hline 22 & Factar (k)Value 0,4 s/d Q7 & 0.700 & data & & & & & & & & & & & & & \\
\hline 23 & Infiltr ation (I = ln. WS) & $\mathrm{Mm} / \mathrm{mth}$ & $(\mathbb{d}) \times(\mathbb{R})$ & ०. प्र & प. पर & 34.56 & 40. 86 & 6.77 & 4418 & ०. प0 & ०. प0 & 409 & ०. प0 & 1208 & 5.12 & \\
\hline 24 & $\mathrm{G}=0.5(1+k) \times 1$ & $\mathrm{~mm} / \mathrm{m}$ th & hitung & ०. प0 & प. पर & 2. 38 & 34.73 & 5. $\pi$ & 37.55 & ०. पा & ०. प0 & 3. 47 & ०. प्र & 107 & 435 & \\
\hline 25 & $L=k \cdot V(n-1)$ & $\mathrm{mm} / \mathrm{mth}$ & Count & 31. 89 & 2232 & 1563 & 3. 50 & 46. 36 & 36.48 & 51. 82 & 36. 8 & T. 39 & ד. 2 & 1414 & 17. 09 & \\
\hline 26 & Volume penyimpanan $\left(V_{n}=\mathrm{G}+\mathrm{L}\right)$ & $\mathrm{mm} / \mathrm{mth}$ & $(\mathrm{Z})+(\mathrm{T})$ & 31. 89 & 2232 & 45. . 0 & 66. $\mathrm{B}$ & 5212 & 74. 03 & 51. 82 & 36. 8 & B. 87 & ד. 2 & 24.41 & 2. 44 & \\
\hline 27 & Changes saved $\left(\Delta V_{n}=\left(V_{n-1}-V_{n}\right)\right.$ & $\mathrm{mm} / \mathrm{mth}$ & Count & 1367 & 9. 57 & -2268 & -2.8 & $14 \|$ & -2.91 & 228 & 1555 & 7.41 & 8. 66 & $-4 . \rrbracket$ & 297 & 24. 12 \\
\hline 28 & Base How $(B F=I+\Delta V n)$ & $\mathrm{mm} / \mathrm{mth}$ & $(\mathbb{Z})+(\mathbb{Z})$ & 1367 & 9. 57 & 11. 80 & 1963 & T. 89 & $22 \pi$ & 221 & 1555 & 11.50 & 8. 66 & 7.87 & 8. ‥ & 17.77 \\
\hline 29 & Direct Runoff (DR = WS - I) & $\mathrm{mm} / \mathrm{m}$ th & (d) - ( (B) & ०. प0 & प. प्र & 34.56 & 40. 86 & 6.77 & 4418 & ०. प0 & ०. प0 & 409 & ०. प्र & 1208 & 5.12 & 147.65 \\
\hline 30 & Runaff (RD = BF + DR) & $\mathrm{mm} / \mathrm{m}$ th & $(\mathbb{B})+(\mathrm{Q})$ & 1367 & 9. 57 & 46. 44 & 60. 49 & 7. 66 & 66. 44 & 221 & 1555 & 1558 & 8. 66 & 1995 & 132 & 319.42 \\
\hline $\mathbf{V}$ & STORM RUNDFF, (SR & & & & & & & & & & & & & & & \\
\hline 31 & Areas ofheavyгain (SR = \%TPN) & $\% \times$ TPN & 0.3 & 28. 18 & $10 \%$ & ०. प्र & प. पर & ०. प0 & ०. प्र & T. 45 & 1890 & ०. प्र & 17.17 & ०. प्र & ०. प्र & 101. 94 \\
\hline 32 & Ex ess Water $(W S \alpha)$ & & Simulation & ०. प0 & ०. (स & 69.12 & 7.48 & 1355 & 59.17 & ०. प0 & ०. प0 & 8.17 & ०. प0 & 24. 16 & 108 & 65. 89 \\
\hline 33 & $\begin{array}{l}\text { Direct Runoff }(D R=W S \alpha-1)+ \\
\text { SR }\end{array}$ & $\mathrm{mm} / \mathrm{mth}$ & Caount & 28. 18 & 108 & 34.56 & 30. 62 & 6. 7 & 1500 & T. 45 & 1890 & 409 & 17.17 & 1208 & 5.12 & 20.18 \\
\hline 34 & Runaff (RD = BF + DR) & $\mathrm{mm} / \mathrm{m}$ th & $(\mathbb{B})+(33)$ & 4285 & 1980 & 46. 44 & 50. T5 & 7. 66 & 37. $\mathrm{B}$ & 48. 6 6 & 34.45 & 1558 & T. 83 & 1995 & 132 & 381.95 \\
\hline 35 & Total Day & & & 31 & 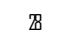 & 31 & 30 & 31 & 30 & 3 & 31 & 30 & 31 & 30 & 31 & \\
\hline 36 & Dis charge of Av alaible & $\mathrm{m}^{3} / \mathrm{sec}$ & $\begin{array}{c}(34) \times(4) \\
/ \mathrm{bl}\end{array}$ & 1967 & 1006 & 2. 32 & B. 84 & $12 \pi$ & 17.67 & 2234 & 1581 & 7. 39 & 11.86 & 9. 46 & 6. 06 & 178.18 \\
\hline $36 \mathrm{a}$ & $\begin{array}{l}\text { Factor of Dischar ge c arr ection } \\
(\beta)\end{array}$ & 1.000 & & 1967 & 1006 & 2. 32 & B. 84 & $12 \pi$ & 17. 67 & 2234 & 1581 & 7. 39 & 11.86 & 9. 46 & 6. 吅 & 178.18 \\
\hline $\begin{array}{l}37 \\
38\end{array}$ & $\begin{array}{l}\text { Initial Deposit }\left(V_{n-1}\right)= \\
\text { SM C }=\end{array}$ & $\begin{array}{l}\mathrm{mm} \\
\mathrm{mm}\end{array}$ & $\begin{array}{c}45.56 \\
186.670\end{array}$ & & & I. & e capa & & & & & & & & & \\
\hline
\end{tabular}

The accuracy of the results of a model needed to test whether the model used to calculate a study can be used in other places with different parameters. The research results are presented in Table 6

Table 6. Comparis on between discharges of model with discharge of observation 


\begin{tabular}{|c|c|c|c|c|c|c|c|}
\hline \multirow{2}{*}{ No } & \multirow{2}{*}{ Year } & \multirow{2}{*}{$\begin{array}{c}\begin{array}{c}\text { Discharge } \\
\text { of Model }\end{array} \\
\left(\mathrm{met}^{3} / \mathrm{sec}\right)\end{array}$} & \multirow{2}{*}{$\begin{array}{c}\text { Discharge of } \\
\text { Observation } \\
\left(\mathrm{met}^{3} / \mathrm{sec}\right)\end{array}$} & \multirow{2}{*}{$\begin{array}{c}\text { Percentage } \\
\text { Storage }\end{array}$} & \multirow{2}{*}{$P=100 m /(n+1)$} & \multicolumn{2}{|c|}{ Ranking } \\
\hline & & & & & & $\begin{array}{c}\text { Discharge of } \\
\text { Model }\end{array}$ & $\begin{array}{l}\text { Discharge of } \\
\text { Observation }\end{array}$ \\
\hline$\overline{\overline{1}}$ & $\overline{2002}$ & 174.85 & $\overline{20.79}$ & $28.58 \%$ & "0.07 & 39.32 & 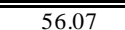 \\
\hline 2 & 2003 & 26.15 & 28.75 & $9.05 \%$ & 0.14 & 33.31 & 42.19 \\
\hline 3 & 2004 & 13.50 & 23.00 & $41.32 \%$ & 0.21 & 32.09 & 34.17 \\
\hline 4 & 2005 & 16.06 & 33.50 & $52.07 \%$ & 0.29 & 26.15 & 33.50 \\
\hline 5 & 2006 & 14.68 & 23.96 & $38.72 \%$ & 0.36 & 19.82 & 30.97 \\
\hline 6 & 2007 & 33.31 & 42.19 & $21.04 \%$ & 0.43 & 18.75 & 28.75 \\
\hline 7 & 2008 & 39.32 & 56.07 & $29.87 \%$ & 0.50 & 16.06 & 28.74 \\
\hline 8 & 2009 & 11.19 & 23.14 & $51.64 \%$ & 0.57 & 14.86 & 26.98 \\
\hline 9 & 2010 & 19.82 & 34.17 & $41.98 \%$ & 0.64 & 14.68 & 26.64 \\
\hline 10 & 2011 & 11.34 & 26.98 & $57.97 \%$ & 0.71 & 13.63 & 23.96 \\
\hline 11 & 2012 & 18.75 & 30.97 & $39.47 \%$ & 0.79 & 13.50 & 23.14 \\
\hline 12 & 2013 & 32.09 & 28.74 & $-11.68 \%$ & 0.86 & 11.64 & 23.00 \\
\hline 13 & 2014 & 15.15 & 26.64 & $43.14 \%$ & 0.93 & 11.34 & 20.79 \\
\hline
\end{tabular}

Graph comparison between the discharge of model with the discharge of observation can be seen in Figure 4 and Figure 5 below:

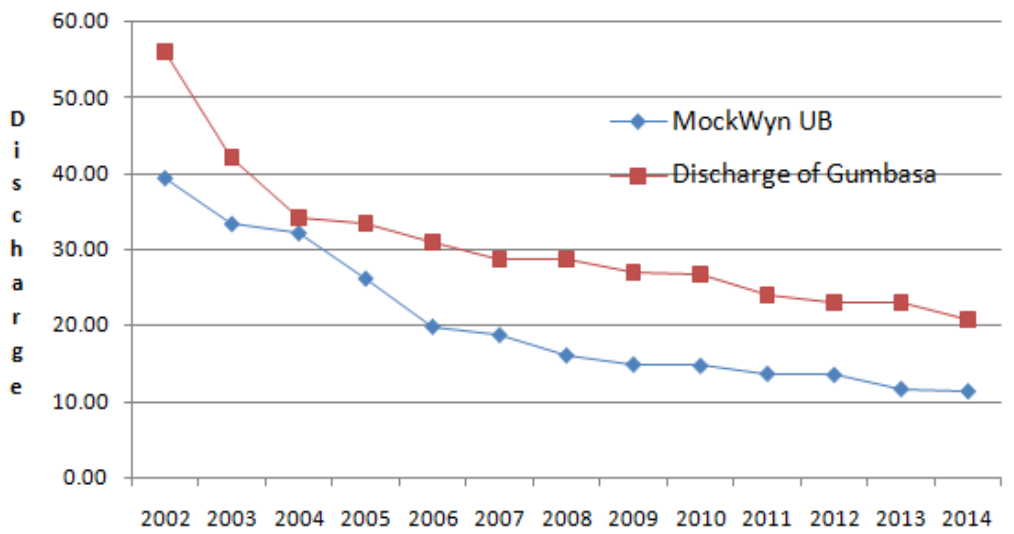

Years of Observation

Figure 4 Graph FDC

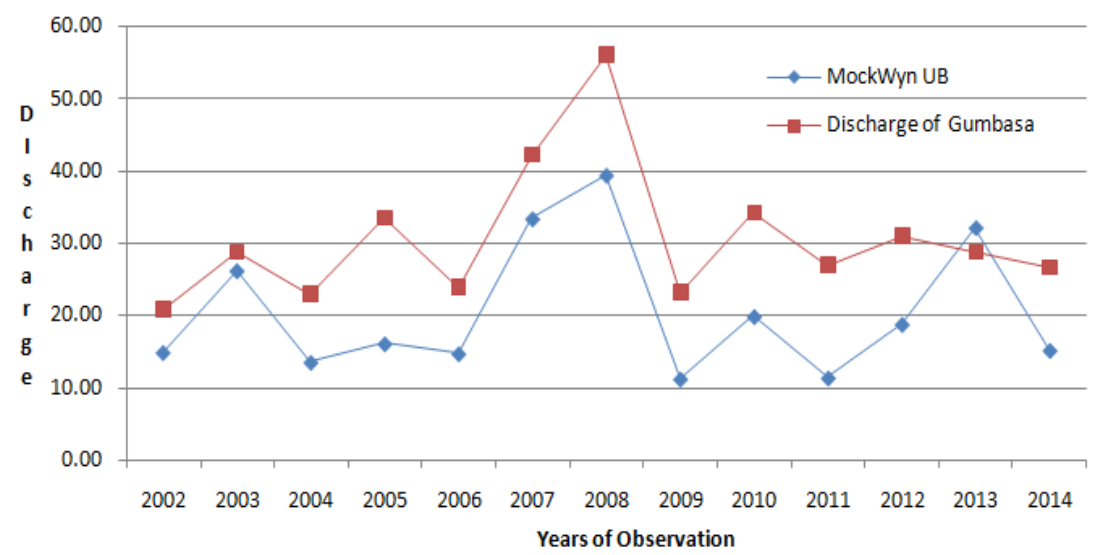

Figure 5. Comparis on between discharge of model with discharge of observation 
Table 7.Parameter of Statistic RMSE

\begin{tabular}{|c|c|c|c|c|c|c|}
\hline No. & Year & $\begin{array}{c}\text { Discharge } \\
\text { of Model } \\
\left(\mathrm{met}^{\mathbf{3}} / \mathrm{sec}\right)\end{array}$ & $\begin{array}{c}\text { Discharge of } \\
\text { FJ Mock } \\
\left(\mathrm{met}^{3} / \mathrm{sec}\right)\end{array}$ & $\begin{array}{c}\text { Discharge of } \\
\text { Obse rvation } \\
\left(\mathrm{met}^{3} / \mathrm{sec}\right)\end{array}$ & $\begin{array}{c}\text { RMSE } \\
\text { MockWyn-UB }\end{array}$ & $\begin{array}{l}\text { RMSE } \\
\text { FJ Mock }\end{array}$ \\
\hline 1 & 2002 & 14.85 & 21.86 & 20.79 & 1.72 & 5.81 \\
\hline 2 & 2003 & 26.15 & 39.80 & 28.75 & 0.75 & 11.27 \\
\hline 3 & 2004 & 13.50 & 15.64 & 23.00 & 2.74 & 3.72 \\
\hline 4 & 2005 & 16.06 & 29.43 & 33.50 & 5.04 & 7.04 \\
\hline 5 & 2006 & 14.68 & 16.69 & 23.96 & 2.68 & 4.05 \\
\hline 6 & 2007 & 33.31 & 48.77 & 42.19 & 2.56 & 13.34 \\
\hline 7 & 2008 & 39.32 & 52.22 & 56.07 & 4.83 & 13.68 \\
\hline 8 & 2009 & 11.19 & 16.17 & 23.14 & 3.45 & 3.67 \\
\hline 9 & 2010 & 19.82 & 23.91 & 34.17 & 4.14 & 5.71 \\
\hline 10 & 2011 & 11.34 & 19.29 & 26.98 & 4.51 & 4.26 \\
\hline 11 & 2012 & 18.75 & 39.45 & 30.97 & 3.53 & 10.37 \\
\hline 12 & 2013 & 32.09 & 56.41 & 28.74 & 0.97 & 16.00 \\
\hline 13 & 2014 & 15.15 & 22.02 & 26.64 & 3.32 & 5.40 \\
\hline \multicolumn{2}{|c|}{ Amount } & 266.21 & 401.66 & 398.91 & 40.24 & 104.33 \\
\hline \multicolumn{2}{|c|}{ Average } & 20.48 & 30.90 & 30.69 & 3.10 & 8.03 \\
\hline
\end{tabular}

Based on the analysis, the relationship between the discharge and the discharge observation results of research in general form a uniform pattern unless there is a difference in 2013. Which debit calculation result is higher than the discharge observation. As for the correlation coefficient between the discharge and the discharge observation models do not qualify so the calibration data using the facility solver is not done. Thus the calibration is done using Root Mean Square Error (RMSE). Value RMSE the calibration stage is obtained $3.10 \%$. While the results of analysis model of Mock at this stage values obtained RMSE $8.03 \%$. The big difference between the discharge and the discharge observation models are affected by several things:

1. Rainfall at the station Kulawi and the station Wuasa greater than other regions. If rainfall is both stations combined percentages exceed the amount of rainfall the three other stations that would affect the calculation of regional rain fall.

2. Existence of Lake Lindu is located in the basin Gumbasa. Lake of Lindu sub-watershed is an area that has $623,35 \mathrm{~km}^{2}$.

\section{CONCLUSION}

Model of discharge MockWyn-UB do not optimal if applied in the watershed Gu mbasa. From the analysis of the percentage deviation between the discharge and the discharge observation models above average $20 \%$. This is caused by the presence of Lindu Lake as a water reservoir so that when it rains, rainwater will be collected first in Lake Lindu, after the water of Lindu Lake full, then the runoff occurs.However the relationship between the discharge and the discharge observation models forming a uniform pattern except in 2013, which is higher than the model discharge observation so as to determine the accuracy of the model discharge test comparisons between models MockWyn-UB model FJ Mock.

Calibration is done by using the Root Mean Square Error (RMSE). MockWyn-UB RMSE value is obtained $3.10 \%$. While the results of model analysis Mock RMSE values obtained $8.03 \%$. From the results above show the RMSE error rate MockWyn-UB models are better than models FJ. Mock.

\section{ACKNOWLEDGEMENTS}

The author would like to thank Sulawesi III River Basin Organization which has provided data on hydrology and climatology and to Satya Wacana has helped to give data Automatic Water Level Recorder (AWLR) Gu mbasa River. 


\section{REFERENCES}

[1]. Mock. F.J, Land Capability Appraisal in Indonesia: Water Availability Appraisal (Bogor: UNDP-FAO Of The United Nations., 1973).

[2]. Crawford, Norman H, and Thurin Steven M, Hydrologic Estimates, National Rural Electric Cooperative Associatio, (Washington, 1981)

[3]. Nugroho.S.P, S. Adi and H. Soewandito.. Influence Change Of Use Against Land Surface Flow, Sediment and Nutrient. Journal of Science and Technology BPPT, 4(5), 2002.

[4]. I Wayan Sutapa, Effect of Climate Change Modelling discharge, Graduate Program Brawijaya University, Malang, 2013.

[5]. Sosrodarsono, Suyono, andTakeda, Hidrology for Irrigation. (Jakarta Publisher Pradnya Paramita, 1983).
[6]. Soemarto C. D, Hidrology Engineering. (Publisher Erlangga-Jakarta, 1995).

[7]. Dunne T, and Leopold L B., 1978. Water in Environtmental Planning (San Fransisco -W.H. Freeman and Company, 1995).

[8]. Monteith J. L, Evaporation and Environment: $19^{\text {th }}$ Symposium of the Society for Experimental Biology, Cambridge Univercity Press, 1965, 205234.

[9]. Ministry of Settlement and Regional Infrastructure, River Flow Forecasting, (Jakarta, 2004).

[10]. Laat P.J.M de, Soil Water-Plant Relations: Lecturer Note, International Institute for Infrastructural, Hydraulic and Enviromental Engineering (IHE), Delf, The Netherlands, 2000. 